\title{
Análisis de residuos sólidos de palma africana, como al- ternativa de aprovechamiento de energías renovables en el departamento del Cesar
}

Analysis of African palm solid waste, as an alternative to use renewable energy in the department of Cesar

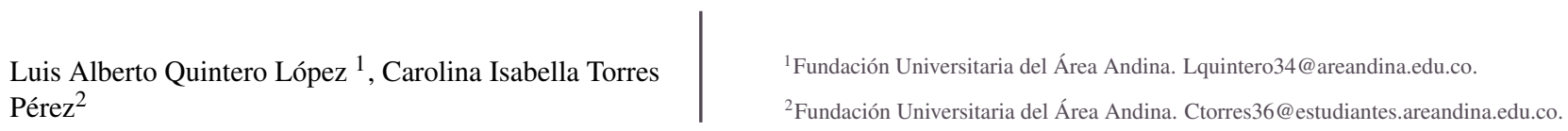

\section{OPEN ACCESS}

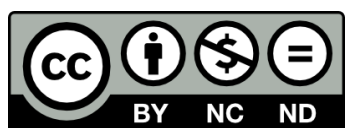

Copyright:(C) 2019 Ingenierías USBmed. La revista Ingenierías USBmed proporciona acceso abierto a todos sus contenidos bajo los términos de la licencia creative commons Atribución- no comercial- SinDerivar 4.0 Internacional (CC BY-NC-ND 4.0)

Tipo de Artículo: Investigación científica y tecnológica.

Recibido: 05-08-2018.

Revisado: 20-01-2019.

Aprobado: 11-02-2019.

Doi: $10.21500 / 20275846.3662$

Referenciar así: L.A., QuinteroLópez.,and C.I., Torrez-Pérez. Y. "Análisis de residuos sólidos de palma africana, como alternativa de aprovechamiento de energías renovables en el departamento del Cesar". Ingenierías USBMed, 10(1), pp.8-18, 2019.

Declaración de disponibilidad de datos: Todos los datos relevantes están dentro del artículo, así como los archivos de soporte de información.

Conflicto de intereses: los autores han declarado que no existen conflicto de intereses.

Editor: Yohana López Rivera, Universidad de San Buenaventura, Medellín, Colombia
Resumen. La demanda actual energética y los costos en energía van incrementando; la contaminación da necesidad a la utilización de energías limpias que impacten en menor medida el medio ambiente. Colombia al encontrarse ubicado en zona rica con climas, biodiversidad y ecosistemas variados, cuenta con gran potencial de energías renovables del aire, agua, sol y biomasa de residuos sólidos como los de palma de africana; hoy en día el $70 \%$ de energía se produce de hidroeléctricas y solo el $2 \%$ del país genera energías renovables. A pesar de que Colombia es un país con gran potencial de biomasa y residuos agroindustriales no se aprovecha su potencial como materia prima en procesos alternos de conversión de energía tales como los procesos termoquímicos. Así mismo el departamento de Cesar cuenta con un potencial de 1.935,51 (TJ/Año) y una generación de residuos anuales de 209.759,99 Ton en 16 Municipios y 8 Plantas de beneficio generadoras de Biomasa residual, que cuentan con un potencial energético de biomasa disponible para su aprovechamiento. De esta manera, es posible establecer por medio de los resultados de investigación el uso de estos residuos en aplicaciones térmicas que conlleven a la generación de energía limpias en la región y el país.

Palabras Clave. Energías renovables; biomasa; residuos agroindustriales; palma de aceite africana.

Abstract. Current energy demand and energy costs are increasing; pollution requires the use of clean energies that impact the environment to a lesser extent. Colombia, being located in a rich area with climates, biodiversity and varied ecosystems, has great potential for renewable energies of air, water, sun and solid waste biomass such as African palm; Nowadays $70 \%$ of energy is produced by hydroelectric power plants and only $2 \%$ of the country generates renewable energy. Although Colombia is a country with great potential for biomass and agroindustrial waste, its potential as a raw material in alternative energy conversion processes such as thermochemical processes is not used. Likewise, the department of Cesar has a potential of 1,935.51 (TJ / year) and a generation of annual waste of 209,759.99 Ton in 16 Municipalities and 8 Biomass waste generation plants, which have an energy potential of biomass available for its use. In this way, it is possible to establish, through research results, the use of these wastes in thermal applications that lead to the generation of clean energy in the region and the country.

Keywords. Renewable energies; biomass; agro-industrial waste; African oil palm. 


\section{Introducción}

Colombia cuenta con una gran biodiversidad y un importante potencial de biomasas, las actividades agroindustriales en el país producen más de 70.000.000 toneladas de residuos agrícolas de cosecha e industriales al año, en cultivos de café, plátano, caña, palma de aceite, maíz, banano y arroz. Colombia se ha posicionado en los últimos años como el primer productor de palma de aceite en América Latina, y el cuarto a nivel mundial, con una producción superior a un millón de toneladas de aceite en 2013, correspondientes a un $1.85 \%$ del total de la producción mundial [1].

La producción de aceite crudo de palma se ha incrementado en un $60 \%$ en el país en los últimos 5 años. Este incremento va acompañado de la producción de cada vez mayores cantidades de residuos generados en el proceso de extracción [2]. En el proceso de beneficio del fruto de palma de aceite se generan varios subproductos de interés técnico y económico; entre ellos: las tusas, la fibra resultante del prensado del fruto, el cuesco obtenido del rompimiento de la nuez, las cenizas producidas por la quema de fibra y cuesco en las calderas [2].

De la biomasa generada se destaca el uso de un alto porcentaje de fibra y cuesco como combustible en la caldera para producción de vapor y electricidad [3]. En el año 2010, Cenipalma realizó un estudio en las plantas de beneficio de las diferentes zonas palmeras de Colombia, concluyendo que del cuesco total obtenido el $67 \%$ se utiliza como combustible para calderas, $16 \%$ se dispone para la venta a otras industrias como combustible, $5 \%$ es utilizado en compostaje, $7 \%$ como acondicionador en las vías de las plantaciones y $5 \%$ en otro usos [4].

Todas estas producciones naturales de los residuos de la palma africana crean una ventaja en el aspecto económico pues consigue beneficios monetarios que repercuten en ahorro de dinero al momento de sostener activamente la planta de beneficio, y con ello hace una producción ambiental más limpia y favorable con el medio ambiente [5]. La investigación se realizó con el fin de estudiar el aprovechamiento de los residuos sólidos de palma de aceite en valores caloríficos de las fibras, cuescos y tusas; los cuales en la industria tienen otros usos y tratamientos que muestran la importancia del residuo sólido como fuente de energías renovables, estos residuos representan entre el $70-74 \%$ de la extracción de palma [6]; así mismo, genera impacto a la comunidad académica como fuente bibliográfica y aporte como proyecto de divulgación y prensa del aprovechamiento energético de los residuos agroindustriales los cuales comprenden una alternativa viable mediante procesos de transformación termoquímica en el departamento del Cesar.

Por lo tanto, es necesario que el departamento del Cesar cuente con planeamientos que converjan al desarrollo energético sustentable y la eficiencia energética desde la evaluación de los recursos renovables, por ello se plantea la oportunidad de cuantificar el poder calorífico de los residuos sólidos del procesamiento de palma africana, y así considerar un aprovechamiento energético óptimo y sustentable de este recurso renovable. Se desarrolló una investigación inicial según los referentes teóricos para identificar las empresas productoras de Biomasa y con ello los procesos de generación de residuos sólidos de palma africana en el departamento del Cesar. Para ello, se analizó la información suministrada por el Departamento administrativo nacional de estadísticas- DANE, Fedepalma, Instituto geográfico Agustín Codazzi - IGAC y unidad de planeación minero energética UPME [7, 8].

\section{Antecedentes}

El uso de la biomasa para la producción de energía ha ganado cada vez más importancia en el escenario mundial y ha estimulado una mayor investigación sobre la biomasa de diferentes fuentes agroindustriales [9]. Los residuos han sido una materia prima esencial en el suministro de energía para muchos sectores incluyendo los agrícola e industrial[10]. El uso racional y adecuado de los residuos como fuente de energía debe ser guiado hacia el aprovechamiento de las propiedades energéticas, entre ellas el poder calorífico que ha sido una de las variables más ampliamente estudiadas para evaluar la calidad de la energía a partir de la producción de biomasa [11].

La transición energética ha sido definida como el proceso que conduce a sustituir y a diversificar las fuentes primarias de energía que son utilizadas en el mundo. Refleja la disponibilidad relativa de los recursos y la economía de su uso, atendiendo sobre todo el precio de mercado y el rendimiento energético, así como factores exógenos como la sustentabilidad ambiental [12]. Así, el proceso de transición debe dirigirse hacia un balance energético más equilibrado, diverso, eficiente y favorable al ambiente, sustituyendo las fuentes energéticas que provienen de recursos fósiles por energías "limpias" [13].

El cambio climático, la generación de energía y la contaminación ambiental son amenazas y desafíos que enfrenta la humanidad en la actualidad [14]. Colombia se ha posicionado en los últimos años como el primer productor de palma de aceite en América Latina, y el cuarto a nivel mundial, con una producción superior a un millón de toneladas de aceite en 2013, de este modo, el departamento del Cesar ubicado al norte de Colombia, se caracteriza por ser una región de tradición agrícola, en 2015 la producción de aceite de palma crudo alcanzó las 281000 toneladas siendo $22 \%$ de la producción Nacional $[4,15]$.

Así mismo, cuenta con 20 municipios palmeros y 10 plantas de beneficio en todo el departamento, con 68.111 hectáreas en producción [16]. De tal modo, en el aprove- 
chamiento de los residuos sólidos de palma de aceite se discuten el valor calorífico de las fibras, cuescos y tusas; los cuales en la industria tienen otros usos y tratamientos que muestran la importancia del residuo solidos como fuente de energías renovables, este incluye la destilación seca para la producción de carbón vegetal. Los análisis que se pueden obtener de estos residuos son el poder calorífico bruto (Btu/lb), Humedad, Materia volátil, Cenizas, Carbono fijo, Azufre, Carbono, Hidrógeno, Nitrógeno, Oxígeno $[17,18]$.

\section{Marco Teórico}

El mundo se va expandiendo y es necesario garantizar la sostenibilidad del medio ambiente frente al crecimiento económico; los combustibles fósiles como el gas natural, el petróleo y el carbón, que se mencionan como las más importantes fuentes de energía en el mundo se agotan día a día y estos recursos no renovables se vuelven raros [19]. Sin embargo, son una fuente favorable de energía; el marco depende de caracterización energética por procesos termoquímicos, fuente de energía renovable biomasa, residuos sólidos de producción de palma de aceite africana [15].

$\mathrm{El}$ aceite de palma es una materia prima importante y versátil para La alimentación y las industrias no alimentarias, es la principal plantación de cultivo en Malasia siendo la fuente más importante de aceite vegetal en el mundo [20]. En el sector de los transportes ha aumentado la demanda de Aceite de palma como materia prima para el transporte de biocombustibles, debido mayores rendimientos y fuerte intención de reducir los gases de efecto invernadero (GEI) $[21,22]$.

Una de las fuentes de energía renovable es la biomasa, la cual es conocido por su alto potencial para satisfacer las necesidades de energía de la sociedad contemporánea, ya sea en desarrollo o industrializados; la biomasa se entiende como el material orgánico no fosilizado y biodegradable procedente de plantas, animales y microorganismos, incluyendo productos, subproductos, residuos y desperdicios de agricultura, silvicultura e industrias relacionadas [23]. La biomasa incluye también los gases y líquidos de la descomposición de no fosilizado y biodegradable material orgánico, es una solución que contribuye a cubrir necesidades energéticas de una forma sostenible [24] (Véase la Figura 1).

En comparación con los combustibles fósiles, la biomasa posee un contenido energético de mayor relación de oxígeno e hidrógeno con respecto a los combustibles fósiles que justamente poseen una mayor cantidad de energía que se almacena en la relación carbono - carbono [7].

Las calderas de combustión de biomasa o combustibles asimilables a esta, hoy día, pueden obtener eficiencias superiores al 86\% sobre PCI (Poder calorífico inferior). Se utilizan presiones de vapor de más de 30 bares y temperaturas del orden de $\operatorname{los} 450^{\circ} \mathrm{C}$ [25].

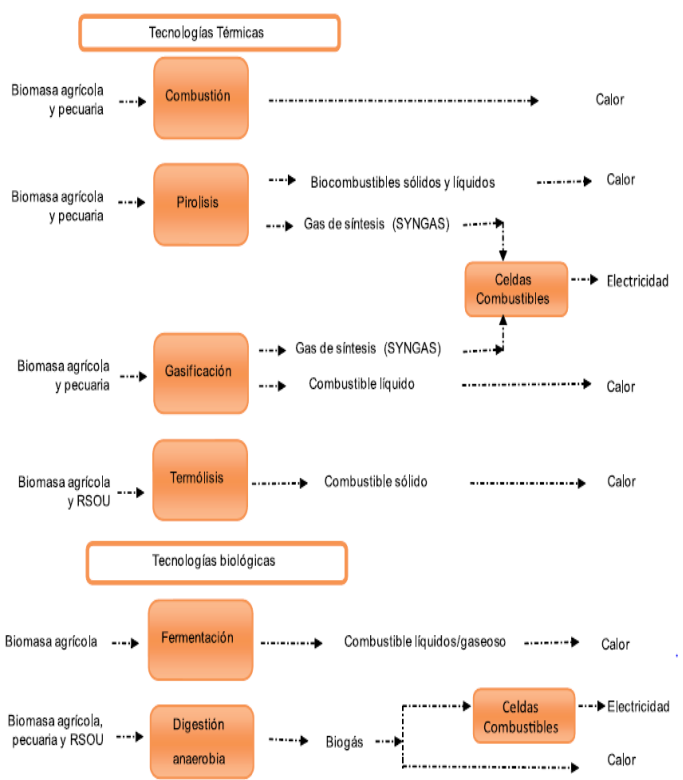

Figura 1. Transformaciones energéticas de biomasa [7].

El proceso más utilizado es el del ciclo Raquis aunque existen instalaciones con gasificación de biomasa para su posterior aprovechamiento en motores de combustión interna. La cantidad de calor desprendido por la combustión completa de la unidad de masa de un cuerpo no depende más que de la composición química del combustible [21] . Esto se ve reflejado en Malasia, donde se han fijado objetivos para el uso de combustibles basados en la biomasa como un combustible renovable alternativo, la principal fuente de combustible son los residuos de palma aceitera, incluyendo racimos vacíos (EFB), conchas y las fibras. En Colombia, los sub-productos de la palma de aceite se utilizan solo parcialmente; la fibra y el cuesco se utilizan para generar vapor de agua para el proceso [26].

Malasia siendo uno de los mayores productores de palma de aceite, está reforzando su producción con el aumento de la demanda mundial de aceite comestible, biodiesel y oleoquimicos derivados del aceite de palma [27]. Por cada $\mathrm{kg}$ de aceite de palma extraído, aproximadamente $4 \mathrm{~kg}$ de biomasa seca generada, de los cuales un tercio se encuentra en los subproductos derivados del racimo de fruta fresca (FFB), la cáscara de la palma (PKS) y el mesocarpio Fibra (MF), (vease la Figura 2) y los otros dos tercios está representado por la palma de aceite Tronco (OPT) y fronda de palma aceitera (OPF) dentro de las plantaciones [28, 29].

Una de las principales características de un biocombustible sólido es su poder calorífico, tanto superior como inferior. El poder calorífico superior (PCS) se define como la energía liberada cuando una masa unitaria de biocombustible se quema con oxígeno en una bomba calorimétrica en condiciones normalizadas [30]. Este PCS, obtenido en laboratorios especializados, permite conocer la energía contenida en la biomasa estudiada incluyendo aquella que se consumirá en evaporar el agua producida en la combustión. 


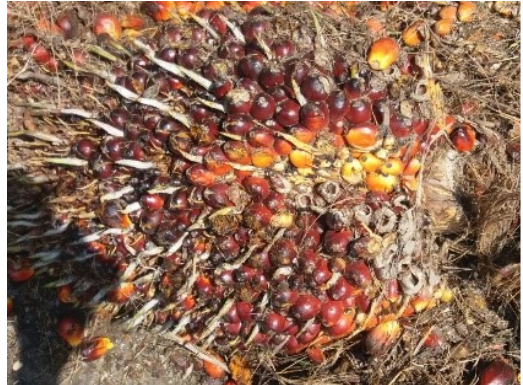

Figura 2. Racimo y fruta de palma de aceite africana.

Sin embargo, la energía realmente aprovechable es aquella que se obtiene una vez evaporada el agua producida durante la combustión. A esta energía se la denomina poder calorífico inferior (PCI) y es necesario utilizar fórmulas empíricas para su determinación a partir del PCS; frente a esto, la determinación de la humedad de la biomasa es fundamental ya que influye en la disminución del poder calorífico y en el aumento del consumo de combustible [31].

Por otro lado, el proceso de compostaje de estos residuos y su conversión en un abono orgánico, tiene el potencial de solucionar la degradación de los suelos, sin dejar de lado la interesante propuesta de rentabilidad económica que justifica su ejecución en escala comercial [32]. De este modo, el compostaje es un proceso de transformación de la materia orgánica para obtener compost, un abono natural. El compostaje proporciona la posibilidad de transformar de una manera segura los residuos orgánicos en insumos para la producción agrícola [33].

La FAO define como compostaje a la mezcla de materia orgánica en descomposición en condiciones aeróbicas que se emplea para mejorar la estructura del suelo y proporcionar nutrientes [34] (vease la Figura 3). Sin embargo, no todos los materiales que han sido transformados aeróbicamente, son considerados compost. El proceso de compostaje incluye diferentes etapas que deben cumplirse para obtener compost de calidad $[35,36]$.

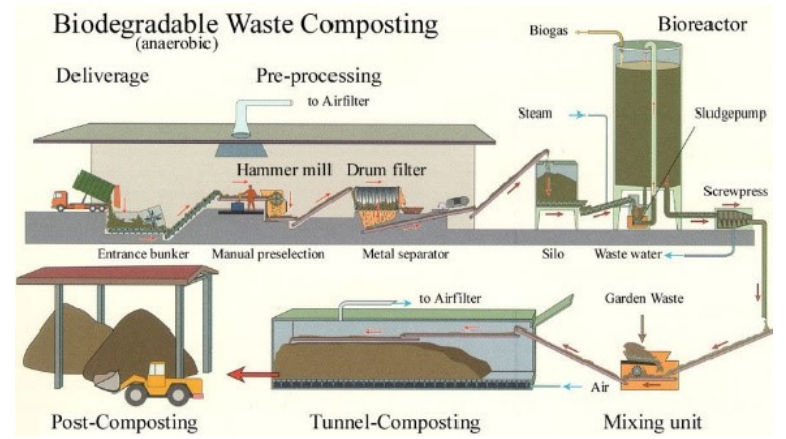

Figura 3. Proceso de digestión anaeróbica y compostaje aeróbico [34].

\section{A. Espacio geográfico de estudio}

Esta investigación se encuentra localizada en el departamento del Cesar (vease la Figura 4) uno de los 32 depar- tamentos de Colombia. Está situado en la zona noreste del país, posee una extensión de $22.905 \mathrm{~km}^{2}$ y una población de 1.041,203 habitantes. Situado en la parte nororiental del país, Cesar limita al norte con los departamentos de La Guajira y Magdalena; por el sur, con Bolívar, Santander y Norte de Santander; y por el este, con Norte de Santander y la República Bolivariana de Venezuela. Sus coordenadas son $07^{\circ} 41^{\prime} 16^{\prime \prime}$ y $10^{\circ} 52$ ' $14^{\prime \prime}$ de latitud norte y $72^{\circ} 53^{\prime} 27^{\prime \prime}$ y $74^{\circ} 08^{\prime} 28^{\prime \prime}$ de longitud oeste. Su capital es Valledupar $[37,38]$.

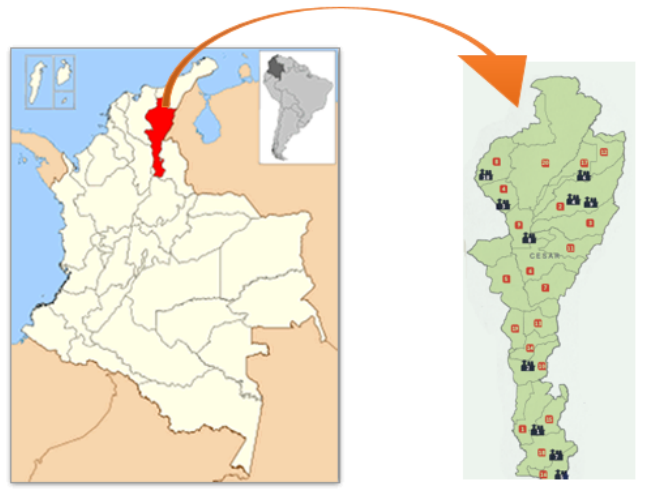

Figura 4. Espacio geográfico de estudio, departamento de Cesar Colombia, con 10 plantas de beneficio palmeras[16].

\section{B. Marco legal y normativo ambiental}

El desarrollo sostenible en Colombia es el que conduzca al crecimiento económico, a la elevación de la calidad de vida y al bienestar social, sin agotar la base de recursos naturales renovables en que se sustenta, ni deteriorar el medio ambiente o el derecho de las generaciones futuras a utilizarlo para la satisfacción de sus propias necesidades $[39,40]$.

El artículo 18. Energía de residuos; se faculta al Ministerio de Minas y Energía para reglamentar normas técnicas que definan los parámetros de calidad que han de cumplir los combustibles sólidos recuperados obtenidos a partir de diferentes residuos. Además, el Ministerio de Ambiente y Desarrollo Sostenible en conjunto con el Ministerio de Agricultura y Desarrollo Rural y el Ministerio de Vivienda, Ciudad y Territorio desarrollarán las estrategias conjuntas para que los combustibles sólidos recuperados que alcancen los parámetros que en dichas normas se consideren necesarios, sean destinados a la valorización energética [41].

Resolución 909 de 2008, expedida por el Ministerio de Medio Ambiente y Desarrollo Sostenible (MADS). Las plantas de beneficio del sector palmero deben cumplir los parámetros de emisiones de fuentes fijas; debido a los estándares para fuentes fijas operantes con biomasa emitidos en esta norma, desde el año 2010 el sector palmero ha participado en un proceso de "Propuesta de Modificación de la Resolución 909 de 2008" con los siguientes antecedentes:

Noviembre de 2010, Fedepalma solicitó un espacio ante 
el MADS, para desarrollar un estudio técnico con el fin de recopilar información del sector. Noviembre de 2011 - I, Se desarrolló el estudio de la viabilidad técnica y económica del cumplimiento de la resolución, el cual contempla el análisis de las emisiones del sector y su afectación a la calidad del aire; asimismo, la modelación de dispersión de los contaminantes emitidos.

Noviembre de 2011-II semestre 2012, Socialización de los resultados obtenidos y concertación de la propuesta de modificación de la norma con las cuatro zonas palmeras del país. I semestre 2013, El MADS abrió un espacio de recepción de observaciones a la norma por parte de los sectores adscritos a la misma. Fedepalma presentó los resultados obtenidos en el estudio técnico y la propuesta de modificación de la norma ante el Grupo de aire de la Oficina de Gestión Ambiental Urbana, y envió dichos documentos al despacho de la actual viceministra Adriana Soto.

El Ministerio de Ambiente y Desarrollo Sostenible inició en el año 2010 el proceso de modificación de la norma de vertimientos puntuales en Colombia. Esta norma tiene como objetivo derogar los actuales estándares de vertimientos del Decreto 1594 de 1984, que aplican a los vertimientos directos de las plantas de beneficio del sector palmero [42].

\section{Metodología}

Para que una investigación establezca un potencial de análisis se identifican los principales factores que lo convierten en oportunidad o en su defecto en una amenaza sobre el patrimonio, los bienes o sobre la población. Es decir, el ser potencial depende del análisis de factores como: Condiciones de bosques de la zona, Disponibilidad de recursos hídricos y energéticos, Facilidad de acceso, Demanda y oferta actual y futura. De esta forma se desarrolló una investigación inicial según los referentes teóricos para identificar las empresas productoras de Biomasa y con ello los procesos de generación de residuos sólidos de palma africana en el departamento del Cesar. Para ello, se analizó la información suministrada por el Departamento administrativo nacional de estadísticas (DANE), Corpoica, Instituto geográfico Agustín Codazzi (IGAC) y unidad de planeación minero-energética UPME [8].

Dentro de la investigación es de tipo cuantitativo, según [43] es un proceso sistemático y ordenado que se lleva a cabo siguiendo determinados pasos, consiste en proyectas el trabajo de acuerdo con una estructura lógica de decisiones y una estrategia que oriente la obtención de respuestas adecuadas a los problemas de indagación propuesto; así mismo un diseño cuasi experimental, en este tipo de investigación nos aproximamos a los resultados de una investigación experimental en las que no es posible el control y manipulación absolutos de las variables [44, 45].

Se entiende como población el conjunto de todos los individuos que aportan información sobre el fenómeno de estudio. La población en esta investigación son los habitantes del departamento del Cesar enfáticamente los 764 palmeros según Censo Nacional de Palma 2011, los más de 26.000 empleos directos e indirectos generados por la producción de palma de aceite en el departamento del Cesar [16].

El proceso se hizo a través una muestra probabilístico, donde los elementos tienen la misma probabilidad de ser escogidos, se hace una selección al azar o aleatoria de los elementos o unidades de muestreo, de este modo, se calcula el tamaño de la muestra en la investigación, ubicando al departamento del Cesar y los 20 municipios palmeros que lo conforman, tiene criterios propios de caracterización energética por procesos termoquímicos, fuente de energía renovable biomasa, residuos sólidos de producción de palma de aceite africana y determinación de poderes caloríficos [46].

Con la información base identificada, se procede a desarrollar las diferentes visitas a las plantas procesadores de palma áfrica para conocer la información primeria necesaria para el desarrollo de la propuesta, y además cuantificar la cantidad de biomasa producida en el departamento y que pueda ser utilizada como fuente de energía renovable tanto para las empresas que la producen como para toda aquella que quiera tener unos procesos de producción más limpios y amigables con el medio ambiente. Con el reconocimiento de biomasa procedente de las plantas procesadoras en el departamento del Cesar, se procederá a realizar una toma de muestra de cada una de la biomasa generada en este proceso (Tusa, Fibra y Cuesco), con el fin de cuantificar por medio de una caracterización de análisis ultimo y próximo el poder calorífico de estos residuos sólidos agroindustriales generados por cada una de estas empresas, y así poder llevar una comparación de lo encontrado bibliográficamente y lo analizado en la etapa práctica de la propuesta de investigación [47].

Por último, con la información de la caracterización podríamos definir cuál es la biomasa idónea para la generación de energía renovable y la potencialidad que podría tener al ser utilizada en diferentes procesos que no solo ayudaría a la eficiencia energética de una empresa, sino que esto se va ver representado en la optimización de procesos, disminución de costos y además se contribuiría con un ambiente más limpio. De igual forma, estos resultados darán pie para abordar futuras investigaciones en líneas de actuación encaminadas a mejorar fundamentalmente al diseño y optimización de sistemas con el objeto de minimizar los costos. 
Tabla 1. Resultados obtenidos atributos potencial energético biomasa por palma de aceite africano en el departamento del Cesar 2017.

\begin{tabular}{lllllll}
\hline \hline $\mathbf{N}^{\circ}$ & Municipio & $\begin{array}{l}\text { Área } \\
\text { sembrada (Ha) }\end{array}$ & $\begin{array}{l}\text { Producción } \\
(t \text { producto/ año) }\end{array}$ & $\begin{array}{l}\text { Rendimiento } \\
(\mathbf{T J} / \mathbf{H a}-\mathbf{a n ̃ o})\end{array}$ & $\begin{array}{l}\text { Cantidad Residuo } \\
(t / \mathbf{a n ̃ o})\end{array}$ & $\begin{array}{l}\text { Potencial energético } \\
(\mathbf{T J} / \mathbf{a n ̃ o})\end{array}$ \\
\hline 1 & Valledupar & 1440,00 & 5040,00 & 3,50 & 9593,64 & 92,54 \\
2 & La Paz & 680,00 & 2448,00 & 3,60 & 4659,77 & 44,95 \\
3 & San Diego & 70,00 & 252,00 & 3,60 & 479,68 & 4.63 \\
4 & Agustín Codazzi & 3620,00 & 7240,00 & 2,00 & 13781,34 & 132.94 \\
5 & Becerril & $2.300,00$ & $8.050,00$ & 3,50 & 15323,17 & 147.81 \\
6 & El $\backslash$ Copey & $7.500,00$ & $25.750,00$ & 3,43 & 49015,13 & 472.82 \\
7 & Bosconia & 1170,00 & 4329,00 & 3,70 & 8240,25 & 79.49 \\
8 & La Jagus $\backslash$ De Ibirico & 400,00 & 1280,00 & 3,20 & 2436,48 & 23.50 \\
9 & Chiriguaná & 600,00 & $2.220,00$ & 3,70 & 4225,77 & 40.76 \\
10 & Curumaní & 100,00 & 320,00 & 3,20 & 609,12 & 5.88 \\
11 & Tamalameque & 925,00 & $2.313,00$ & 2,50 & $4.402,80$ & 42.47 \\
12 & Pelaya & 145,00 & 363,00 & 2,50 & 690,97 & 6.67 \\
13 & Aguachica & 650,00 & $2.600,00$ & 4,00 & $4.949,10$ & 47.74 \\
14 & Río De Oro & 880,00 & $3.344,00$ & 3,80 & $6.365,30$ & 61.40 \\
15 & San Martín & $4.850,00$ & $12.600,00$ & 2,60 & $23.984,10$ & 231.36 \\
16 & San Alberto & 8500,00 & 32300,00 & 3,80 & 61483,05 & 593.09 \\
\hline \hline
\end{tabular}

\section{Resultados y análisis}

El proceso de extracción de aceite crudo de palma genera subproductos como son el raquis de palma, la fibra, el cuesco, lodos de piscinas de oxidación y cenizas, conocidos como residuos agroindustriales, (véase la Figura 5); los cuales los tres primeros son residuos sólidos aprovechables si son canalizados y manejados adecuadamente, ya que son una fuente potencial de ingresos para las plantas y resuelven un problema ambiental de disposición de los mismos [48].

Durante los análisis de la importancia económica de residuos con producciones destacadas en cantidad y disposición del producto a través del establecimiento geográfico de las plantas en la zona de estudio, se obtuvo una amplia base de datos de plantas productora de residuos de palma de aceite, seleccionadas a través del impacto que tienen en la región y por la cantidad de subproductos que proveen.

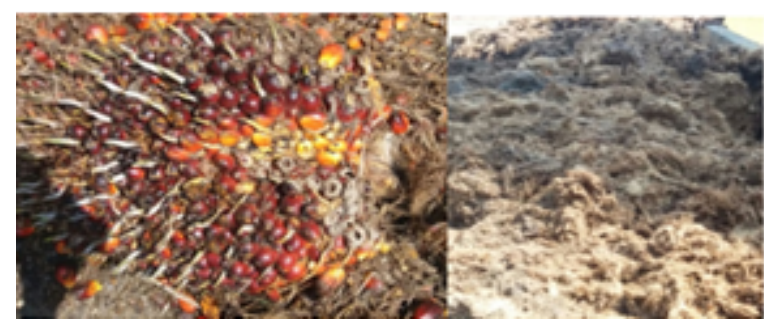

Figura 5. Residuos agroindustriales, fibra y cuesco de palma de aceite 2017

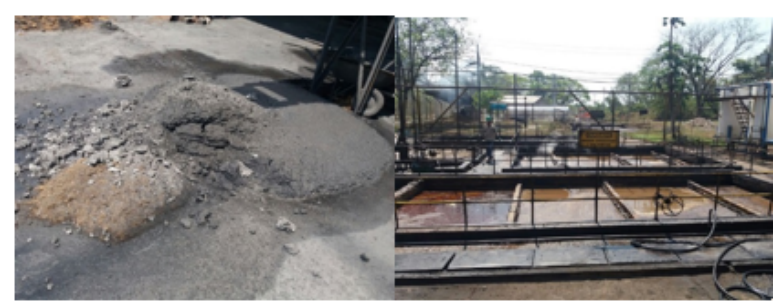

Figura 6. Residuos agroindustriales, Cenizas y lodos de oxidación de palma de aceite 2017.

\section{A. Biomasa en el Departamento del Cesar}

Se realizó una delimitación cartográfica en el software arcgis del atlas de biomasa en Colombia, (véase Figura 7) especificando el departamento del Cesar, área de estudio, las plantas de beneficio de palma de aceite africana presentes y generando tablas de atributos, gráficas de porcentaje e histogramas del potencial energético por municipios. (Véase Tabla 1).

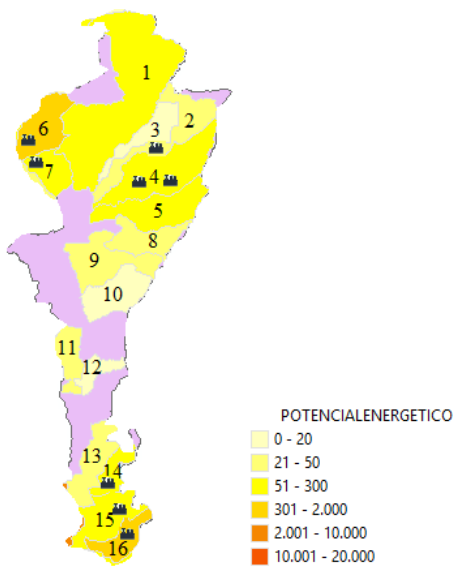

Figura 7. Mapa de Biomasa departamento del Cesar, potencial palma de aceite TJ por año. Adaptado en SIG Arcgis 2017. 
Tabla 2. Plantas de beneficio en el Departamento del Cesar con potencial energético de biomasa por palma de aceite africana. 2017. Adaptado por Carolina Torres.

\begin{tabular}{llll}
\hline \hline $\mathbf{N}^{\circ}$ & MunicipioPlanta de Beneficio & Municipios & Potencial energético (TJ/año) \\
\hline 2 & Oleoflores S.A. & La Paz & 44,95 \\
4 & Extractora Sicarare S.A.S. & Agustín Codazzi & 132,94 \\
4 & Palmas Oleaginosas de Casacara Ltda., Palmacara & Agustín Codazzi & 132,94 \\
6 & Palmeros de la Costa S.A. & El Copey & 472,82 \\
7 & Extractora Palmariguaní S.A. & Bosconia & 79,49 \\
14 & Agroindustrias del Sur del Cesar Ltda. y Cía., & Agroince Rio de Oro & 61,40 \\
15 & Palmas del Cesar S.A. & San Martin & 231,36 \\
16 & Industrial Agraria La Palma Ltda., Indupalma Ltda. & San Alberto & 593,09 \\
\hline \hline
\end{tabular}

Se determina (véase la Tabla 1) que el departamento del Cesar cuenta con un alto potencial energético de biomasa residual en palma de aceite africana 1935.51 (TJ/Año) y una generación de residuos anuales de 209.759,99 Ton, siendo los municipios de El Copey y San Alberto con mayor Ha sembradas, mayor potencial energético y un rendimiento de 3.43-3.80. Además, los Municipios de San Martin y Agustín Codazzi que también cuentan con un gran número de hectáreas sembradas son los que cuentan con menor rendimiento, pero significativo poder energético de biomasa residual.

Por consiguiente, la producción de biomasa por palma de aceite africana tiene un futuro prometedor en el Departamento del Cesar, ya que genera una gran cantidad de residuos agroindustriales los cuales pueden ser utilizados como generadores de energía, un ejemplo a seguir es de la Empresa Palmeras de Costa S.A ubicada en El Copey, quien genera 49.015,13 Ton/ anual de residuos agroindustriales, la empresa utiliza dichos residuos en la producción de compos como fertilizantes para su plantación, generación de energía en su planta y como combustible para calderas; a pesar de su arduo trabajo no utilizan el $100 \%$ la cantidad de residuos que generan [49].

El departamento del Cesar cuenta con 10 plantas de beneficio según informe de Fedepalma 2016, (véase la Tabla 2) se aprecian 8 Empresas, Municipios y el potencial energético de biomasa con el cuentan, de acuerdo a una visita realizada a Oleoflores S.A se determinó que cuenta con sistemas más tecnificados y mayor economía del departamento, pero observamos su poco aprovechamiento de biomasa por el poco potencial energético y gran cantidad de residuos agroindustriales que utilizan en las calderas pero aún les sobran demasiados residuos que no le dan ningún uso [16].

Sin embargo, el departamento del Cesar también cuenta con Palmagro S.A ubicada en el Paso y Extractora la Gloria S.A ubicada en la gloria de estas dos plantas de beneficio se desconoce su potencial energético de biomasa residual.
B. Procesos de generación de residuos solidos

En las calderas se utiliza solo fibra y cáscara, se tiene generación de vapor con una caldera de alta presión acuotubular con una capacidad de 20 toneladas de vapor de las cuales 18 son para el proceso lo cual indica que están muy bien de vapor en todos los procesos de separación se gasta suficiente vapor para la cocción de la fruta, entonces el $90 \%$ de la actividad de la planta es con vapor [50].

C. Cuantificación de la cantidad de fibra y cuesco que ingresa a la caldera

En palmeras de la costa S.A en el año 2017, al dia se estan produciendo $942 \mathrm{KW}$ con producción de 32 ton de vapor; manejan una caldera de biomasa, la cual trabaja con cascara y fibra, el mayor poder calorífico lo tienen las cascaras, pero no pasan de 1 a 1.5 ton y se mete mayor volumen de fibra entre 6 y 7 ton por hora [50]. En la Tabla 3 se evidencia la producción de vapor de acuerdo al tonelaje de RFF y producción de electricidad por tonelaje de residuos de biomasa.

\section{Caracterización de fibra y cascarilla. Labora- torio}

Para el aprovechamiento energético de la Biomasa, se debe caracterizar por tener un bajo contenido de carbono, un elevado contenido de oxígeno y compuestos volátiles (formados por cadenas largas del tipo $\mathrm{CnHm}$, y presencia de $\mathrm{CO} 2, \mathrm{CO}$ e $\mathrm{H} 2$ ) son los que concentran una gran parte del poder calórico de la biomasa, haciéndola más óptima [51].

En general se puede considerar que el poder calórico de la biomasa puede oscilar entre los 3000 - $3500 \mathrm{kcal} / \mathrm{kg}$ para los residuos lignocelulósicos [25], ahora bien de acuerdo a los análisis próximos realizados (véase la Tabla 4) (véase la Tabla 5) la cascarilla tiene un poder calorífico 4276,655 $\mathrm{kcal} / \mathrm{kg}$, azufre $5 \%$ y la fibra $2906,89 \mathrm{kcal} / \mathrm{kg}$, azufre $10 \%$, se determina que es la cascarilla (cuesco) quien cuenta con mayor poder calorífico y menor contenido de azufre, pero 
Tabla 3. Cuantificación de fibra y cuesco para producción de electricidad. Palmeras de la Costa S.A 2017.

\begin{tabular}{|c|c|c|c|c|c|}
\hline \multirow[t]{5}{*}{$\begin{array}{l}\text { Producción de vapor } \\
\text { estimada }\end{array}$} & $\begin{array}{l}\text { Fibra }+ \text { cascaras }+ \\
30 \% \text { raquis }\end{array}$ & 21.42 & 24.99 & 27.13 & 28.56 \\
\hline & Fibra+Cascaras & 17.87 & 20.85 & 22.64 & 23.83 \\
\hline & Cascaras & 6.92 & 8.07 & 8.77 & 9.23 \\
\hline & Fibras & 10.93 & 12.75 & 13.84 & 14.57 \\
\hline & Tusas & 10.67 & 12.45 & 13.52 & 14.23 \\
\hline \multirow{6}{*}{$\begin{array}{l}\text { Disponibilidad de } \\
\text { biomasa para } \\
\text { generación }\end{array}$} & $\begin{array}{l}\text { Fibra }+ \text { cascaras }+ \\
30 \% \text { raquis }\end{array}$ & 7.43 & 8.67 & 9.41 & 9.91 \\
\hline & Fibra+Cascaras & 5.21 & 5.21 & 6.60 & 6.95 \\
\hline & Cascaras & 1.53 & 1.79 & 1.94 & 2.04 \\
\hline & Fibras & 3.68 & 4.29 & 4.66 & 4.91 \\
\hline & Tusas & 6.75 & 7.88 & 8.55 & 9.00 \\
\hline & Producción de RFF (Ton/h) & 3035 & 38 & 40 & \\
\hline \multicolumn{6}{|c|}{$\begin{array}{l}\text { Producción de energía } 23.8 \\
\text { Kg/KW Turbo Shinko) }\end{array}$} \\
\hline \multirow[t]{5}{*}{$\begin{array}{l}\text { Producción de } \\
\text { electricidad hora } \mathrm{KW}\end{array}$} & $\begin{array}{l}\text { Fibra }+ \text { cascaras }+ \\
30 \% \text { raquis }\end{array}$ & 893 & 1042 & 1131 & 1191 \\
\hline & Fibra+Cascaras & 744 & 868 & 942 & 992 \\
\hline & Cascaras & 288 & 336 & 365 & 384 \\
\hline & Fibras & 456 & 532 & 578 & 608 \\
\hline & Tusas & 445 & 519 & 564 & 593 \\
\hline \multirow[t]{6}{*}{$\begin{array}{l}\text { Potencia de generación } \\
\text { ton vap/hr }\end{array}$} & $\begin{array}{l}\text { Fibra }+ \text { cascaras }+ \\
30 \% \text { raquis }\end{array}$ & 21.42 & 24.99 & 27.13 & 28.56 \\
\hline & Fibra+Cascaras & 17.85 & 20.83 & 22.61 & 23.8 \\
\hline & Cascaras & 6.92 & 8.07 & 8.77 & 9.23 \\
\hline & Fibras & 10.93 & 12.75 & 13.84 & 14.57 \\
\hline & Tusas & 10.67 & 12.45 & 13.52 & 14.57 \\
\hline & Producción de RFF (Ton/h) & 30 & 35 & 38 & 40 \\
\hline
\end{tabular}

ambos son óptimos. Estas características, juntamente con el bajo contenido de azufre de la biomasa, la convierten en un producto especialmente atractivo para ser aprovechado energéticamente.

\section{Conclusiones}

Tomando como base los resultados obtenidos de este artículo, se plantean las siguientes conclusiones:

En Colombia existen gran potencial energético de residuos agroindustriales de la palma de aceite (RAI), estos tipos de biomasa son los residuos de cuesco y raquis en las plantaciones, se pueden utilizar para la generación de energía y sustitución de combustibles para las Empresas; el Departamento del Cesar ubicado al norte del País cuenta con múltiples cultivos, producción y tratamiento de palma de aceite, generando gran cantidad de residuos que son aprovechados como fertilizantes en sus cultivos, producción de vapor en calderas y biomasa residual, sin embargo sigue sobrando gran cantidad de residuo agroindustrial.

Según, la caracterización de fibra y cuesco se determinó que el cuesco (cascarilla) cuenta con mayor poder calorífico, menor humedad, menos contenido de azufre; siendo la cascarilla la más óptima para transformación de energías renovables, pero los dos residuos son aprovechables.
Para la ejecución del proyecto se utilizó el software arcgis, analizando la disposición de residuos sólidos del proceso de extracción de palma de aceite en el atlas de biomasa, se determinó que el departamento del Cesar cuenta con un potencial de Biomasa de 1935.51 TJ/año. Con esto se definió que el departamento cuenta con 16 Municipios de aprovechamiento potencial energético de biomasa, 8 Plantas de beneficio generadoras de Biomasa residual que se encuentran distribuidas a lo largo de toda la región, siendo el Municipio de San Alberto con un mayor potencial energético de biomasa de $34 \%$ y a su vez cuenta con la Empresa Industrial Agraria La Palma Ltda.

La utilización de biomasa con fines energéticos posee numerosas ventajas, no solo para el propietario de la instalación, sino también para el conjunto de la sociedad, entre las que destacan; disminución de las emisiones de $\mathrm{CO} 2$ respecto a otros combustibles fósiles, No emite contaminantes sulfurados o nitrogenados (causantes de la lluvia ácida), una gran problemática que presentan todas las empresas es el exceso de residuos, gracias al reciclaje de la biomasa se disminuyen los residuos agroindustriales esto contribuye a la diversificación energética del País, los cultivos energéticos no atentan contra la seguridad alimentaria presentando nuevas oportunidades para el sector agrícola. 
Tabla 4. Reporte de análisis de biomasa Cascarilla de palma de aceite africana, con humedad de 10.09. 2017

\begin{tabular}{llll}
\hline \hline & Análisis próximo complete cascarilla & \\
\hline & Base como se recibe & Base seca & Norma - Método \\
\hline Humedad total \% en peso & 11,54 & 1,90 & ASTM D33021D3302M-12 \\
Ceniza \% en peso & 1,68 & 77,23 & ASTM D 7582 - /12- 3174 - 12 \\
Material Volátil \% en peso & 68,32 & 20,87 & ASTM D 7582 - 12/3175 - 11 \\
Carbono fijo \% en peso & 18,46 & 0,05 & ASTM D3172-07a \\
Azufre \% en peso & 0,05 & 9408 & ASTM D5965-12 \\
Poder calorífico bruto (Btu/Lb) & 8322 & 5227 & ASTM D5965-12 \\
Poder calorífico bruto (Kcal/Kg) & 4623 & 21,88 & ASTM D5965-12 \\
Poder calorífico bruto (GJ/Ton) & 19,36 & & \\
\hline & Poder calorífico neto & \\
\hline Poder calorífico neto a presión constante (BTU/Lb) & 7697,97 & 8839,64 & ASTM D5965-12 \\
Poder calorífico neto a presión constante (GJTM) & 1791 & 20,56 & ASTM D5965-12 \\
Poder calorífico neto a presión constante (J/g) & 17905,48 & 20561,00 & ASTM D5965-12 \\
Poder calorífico neto a presión constante (Kcal/Kg) & 4276,655 & 4910,91 & ASTM D5965-12 \\
\hline \hline
\end{tabular}

Tabla 5. Reporte de análisis de biomasa fibra de palma de aceite africana, con humedad de 36.77

\begin{tabular}{|c|c|c|c|}
\hline \multicolumn{4}{|c|}{ Análisis próximo complete fibra } \\
\hline & Base como se recibe & Base seca & Norma-Método \\
\hline Humedad total \% en peso & 35,44 & & ASTM D33021D3302M-12 \\
\hline Material Volátil \% en peso & 47,84 & 74,09 & ASTM D $7582-12 / 3175-11$ \\
\hline Carbono fijo $\%$ en peso & 1261 & 1955 & ASTM D3172-07a \\
\hline Azufre $\%$ en peso & 0,10 & 0,15 & ASTM D4239-12 Method A \\
\hline Poder calorífico bruto $(\mathrm{Kcal} / \mathrm{Kg})$ & 3327 & 5154 & ASTM D5965-12 \\
\hline Poder calorífico bruto (GJ/Ton) & 13,93 & 21,58 & ASTM D5965-12 \\
\hline \multicolumn{4}{|c|}{ Poder calorífico } \\
\hline Poder calorífico neto a presión constante (BTU/Lb) & 5232,40 & 8680,46 & ASTM D5965-12 \\
\hline Poder calorífico neto a presión constante (GJTM) & 12,17 & 20,19 & ASTM D5965-12 \\
\hline Poder calorífico neto a presión constante (J/g) & 1217057 & 20190,74 & ASTM D5965-12 \\
\hline
\end{tabular}

\section{Referencias}

[1] M. R. Millán, M. A. C. Domínguez, and F. E. S. Vargas, "Efecto de la temperatura en el potencial de aprovechamiento energético de los productos de la pirólisis del cuesco de palma," Tecnura: Tecnología y Cultura Afirmando el Conocimiento, vol. 20, no. 48, pp. 89-99, 2016.

[2] D. F. Flórez Ramos, J. Barco Burgos, and S. L. Rincón Prat, "Análisis comparativo de la carbonización de cuesco de palma de aceite en reactores de lecho fijo," Tecnura, vol. 20, no. 49, pp. 45-58, 2016.

[3] O. A. Santana, "La edad mínima para talar especies nativas con potencial energético en la región semiarida de brasil," NRC Research Press, pp. 411-417, 2017.

[4] J. F. Pérez, R. Barrera, and G. Ramírez, "Integración de plantaciones forestales comerciales colombianas en conceptos de biorrefinería termoquímica: una revisión," Colombia forestal, vol. 18, no. 2, pp. 273-294, 2015.

[5] N. M. B. G. M. Arias, "Posibilidades para el desarrollo sostenible de la palmicultura en mexico: Experiencias y retos.," Spanish Journal of Rural Development, vol. 3, no. 4, pp. 79-88, 2012.

[6] Cenipalma, "Palmeras de colombia," 2010.

[7] U. UPME, IDEAM, "Atlas del potencial energético de la biomasa residual en colombia," 2014.

[8] M. M. Aguilera-Díaz, "Palma africana en la costa caribe: un semillero de empresas solidarias," Capítulo 3. Palma africana en la Costa Caribe: un semillero de empresas solidarias. Pág.: 102-143, 2002.

[9] C. Martel and L. Cairampoma, "Cuantificación del carbono almacenado en formaciones vegetales amazónicas en" cicra", madre de dios (perú)," Ecología Aplicada, vol. 11, no. 2, pp. 59-65, 2012.

[10] P. López and L. Calvo, "Certificación forestal fsc en montes productivos de pequeños-medianos propietarios forestales del norte de españa a partir del estudio de casos de diferentes áreas forestales con predominio de plantaciones en galicia.," Spanish Journal of Rural Development, vol. 2, no. 3, 2011.

[11] R. Bjorheden, "Desarrollo de bioenergía a partir de 
biomasa forestal: un estudio de caso de suecia y finlandia," Croata de Ingeniería Forestal, vol. 38, no. 2, 2017.

[12] V. Durán, C. Rodríguez, J. Francia, S. Sayadi, J. Jiménez, and F. Perea, "Biomasa de cultivos leñosos de rotación corta para la producción sostenible de energía: oportunidades y retos.," Spanish Journal of $R u$ ral Development, vol. 5, no. 1, 2014.

[13] B. I. de Desarrollo et al., "Integración de las energías renovables no convencionales en colombia," 2015.

[14] G. E. Kindermann, S. Schörghuber, T. Linkosalo, A. Sanchez, W. Rammer, R. Seidl, and M. J. Lexer, "Potential stocks and increments of woody biomass in the european union under different management and climate scenarios," Carbon balance and management, vol. 8, no. 1, p. 2, 2013.

[15] D. T. R. H. M. R. Y. D. Rivera-Méndez, "Huella de carbono de la producción de racimos de fruta fresca de palma aceitera ( elaeis guineensis ) en colombia,", ELSEVIER, vol. 149, pp. 743-750.

[16] O. Comunicaciones, "La palma de aceite en el departamento del cesar," Boletín El Palmicultor, no. 530, p. 30, 2016.

[17] R. B. Zapata, J. F. P. Bayer, and C. S. Jiménez, "Carbones colombianos: clasificación y caracterización termoquímica para aplicaciones energéticas," Revista ION, vol. 27, no. 2, 2014.

[18] L. M. T. A. and S. C. Bennamoun, "Evaluación del efecto de la humedad en la simulación de la estrategia de la cadena de suministro de biomasa forestal: estudio de caso de la nueva brunswick, canadá.," Revista Croata de Ingeniería Forestal, vol. 38, no. 1, 2017.

[19] S. E. Hosseini, "Estudio de viabilidad de la producción y utilización de biogás como fuente de energía renovable en malasia.," ScienceDirect, pp. 454-462, 2013.

[20] S. E. Hosseini, "Utilización de residuos sólidos de palma como fuente de energía renovable y sostenible en malasia.," ScienceDirect, 2014.

[21] S.-K. R. S.-H. Kong, "Biochar de la biomasa de palma aceitera: una revisión de su potencial y desafíos," $R e$ newable and Sustainable Energy Reviews, vol. 39, pp. 729-739, 2014.

[22] J. M. Gómez, "Análisis de la variación de la eficiencia en la producción de biocombustibles en américa latina," Estudios Gerenciales, vol. 32, no. 139, pp. 120-126, 2016.

[23] M. Gutiérrez and J. Serna, "Jatropha curcas, una planta que impulsa motores," Revista Universidad EAFIT, vol. 50, no. 165, p. 22, 2015.

[24] O. A. Ingrid Miranda, "Aprovechamiento del potencial energético de la biomasa residual de la extracción del aceite de palma en colombia,," 2009.

[25] L. E. R. V. H. C. A. Cardona, "Análisis de esquemas tecnológicos para la producción eficiente de productos de valor agregado de materias primas oleoquímicas colombianas,", ELSEVIER, vol. 49, no. 3, pp. 474-489, 2014.

[26] M. H. M. Ashnani, "Una fuente de energía renovable, ¿por que el biodisel?,' ScienceDirect,, pp. 244-257, 2014.

[27] Afrizal, "Intervención de terceros para terminar los conflictos de plantaciones de palma aceitera en indonesia: un análisis estructural.," Revista de problemas sociales en el sudeste asiático, vol. 30, no. 1, 2015.

[28] S. Acevedo, L. Giraldo, and J. C. Moreno, "Caracterización textural y química de carbones activados preparados a partir de cuesco de palma africana (elaeis guineensis) por activación química con cacl2 y mgcl2," Revista Colombiana de Química, vol. 44, no. 3, pp. 1824, 2015.

[29] Z. Peck YeanGan, "Estudio econométrico sobre la posición del aceite de palma en malasia mercado mundial hasta 2035," ELSEVIER, vol. 39, pp. 740-747, 2014.

[30] J. C. ARTEAGA, E. ARENAS, A. L. R. DAVID, and Z. ZAPATA, "Obtención de biocombustibles producto de la pirolisis rápida de residuos de palma africana (elaeis guineensis jacq.) obtenção de bicombustíveis por pirólise rápida de resíduos de palma de dendê (elaeisg uineensis jacq.) biofuels production by fast pyrolysis of palm oil wastes (elaeis guineensis jacq.)," Biotecnología en el Sector Agropecuario y Agroindustrial, vol. 10, no. 2, pp. 144-151, 2012.

[31] I. para diversificación y ahorro de la energía, "Manual de energías renovables," 2007.

[32] R. Torres, Á. Acosta, and C. Chinchilla, "Proyecto comercial de compostaje de los desechos agroindustriales de la palma aceitera," Revista Palmas, vol. 25, no. especial,, pp. 377-387, 2004.

[33]

[34] . E. MateusHenriqueRocha, R., "Evaluación del ciclo (acv) de los biocombustibles en condiciones: un meta análisis," ELSEVIER, vol. 37, pp. 435-459, 2014.

[35] O. de las naciones unidas para la alimentación y la agricultura, "Manual del compostaje del agricultor," 2013.

[36] T. G. Castañeda and H. M. Romero, "Compostaje de subproductos de la agroindustria de palma de aceite en colombia: estado del arte y perspectivas de investigación," Boletines técnicos, no. 31, 2012.

[37] G. del Cesar, "Plan estratégico regional de ciencia, tecnología e innovación -percti- departamento del cesar," 2011.

[38] MINAMBIENTE, "Plan integral de gestión de cambio climático territorial del cesar 2032," MINAMBIENTE, Valledupar, 2016, 2016.

[39] L. . general Ambiental de Colombia, "Ley 99 general ambiental de colombia," 1993. 
[40] H. J. G. Álvarez and D. T. Rodríguez, "Conservación de la biodiversidad en colombia, una reflexión para una meta: conocer y educar para conservar," Cuadernos de biodiversidad, no. 42, pp. 31-37, 2013.

[41] p. m. d. 1. c. s. r. 1. i. d. 1. e. r. n. c. a. s. e. Ley 1715 , "Ley 1715, por medio de la cual se regula la integración de las energías renovables no convencinales al sistema energético.,", 2014.

[42] Fedepalma, "Normatividad ambiental," 2017.

[43] C. Monje, "Metodología de la investigación cuantitativa y cualitativa," Neiva: universidad surcolombiana, p. 113, 2011.

[44] C. Ruiz, "Alcance de la investigación cuantitativa," 2010.

[45] C. Gregorio, "Procedimientos de investigación científica," 1986.

[46] J. C. Sanchez, "Los métodos de investigación," Ediciones Diaz de Santos, 2012.

[47] Cenipalma, "Experiencias en el procesamiento industrial de racimos de fruta fresca de la palma de aceite alto oleico interespecíficos oxg (e. oleífera $\mathrm{x}$ e. guineensis)," 2013.

[48] B. Ishani Mukherjee, "Biocombustibles a base de aceite de palma y sostenibilidad en el sudeste asiático: Una revisión de indonesia, malasia y tailandia," ELSEVIER, vol. 37, no. especial, pp. 1-12, 2014.

[49] Fedepalma, "Informe de gestión," 2016.

[50] W. Silva, "Cálculo de procesos, palmeras de la costa," Copey, 2017.

[51] M. A. W. S. E. Hosseini, "Utilización de residuos sólidos de palma como fuente de energía renovable y energía sostenible en malasia,", ELSEVIER, vol. 40, pp. 621-632, 2014. 